\title{
MicroRNA-147 suppresses proliferation, invasion and migration through the AKT/mTOR signaling pathway in breast cancer
}

\author{
YULONG ZHANG ${ }^{1}$, HE ZHANG ${ }^{2}$ and $\mathrm{ZHE} \mathrm{LIU}^{1}$ \\ ${ }^{1}$ Oncology Center, West China Hospital, Chengdu, Sichuan 610041; \\ ${ }^{2}$ Graduate School, Peking Union Medical College and Chinese Academy of Medical Sciences, \\ National Center for Clinical Laboratories, Beijing 100730, P.R. China
}

Received October 17, 2014; Accepted September 21, 2015

DOI: $10.3892 / \mathrm{ol} .2015 .3842$

\begin{abstract}
The Akt/mTOR pathway is considered to be the most frequently aberrantly activated pathway in human tumors. It is activated in a variety of types of tumor, and is therefore an attractive target for study, including it's potential regulation by microRNAs. A number of microRNAs (miRs) have been demonstrated to target the Akt/mTOR pathway. A previous study reported that miR-147 targets the EGFR-driven cell-cycle protein network in breast cancer. EGFR serves a crucial role upstream to Akt/mTOR. To define the function and mechanism of miR-147 in breast cancer, the present study assessed miR-147 expression in a normal mammary epithelial cell line and three breast cancer cell lines, and observed that miR-147 expression was markedly low in the highly invasive cell line, MDA-MB-231. Ectopic expression of miR-147 in MDA-MB-231 resulted in a reduction of the phosphorylation of crucial molecules in the Akt/mTOR pathway and the proliferation, invasion and migration of the cell line was also reduced. The effects of miR-147 expression are similar to that of shRNA which is specifically designed to silence the expression of Akt. The findings of the present study indicate that miR-147 suppressed the proliferation, invasion and migration of breast cancer cells through targeting the Akt/mTOR signaling pathway. As a new microRNA targeting Akt/mTOR pathway. Using miR-147 may therefore provide an effective therapeutic approach to suppress tumorigenicity in breast cancer.
\end{abstract}

\section{Introduction}

Breast cancer is a malignant disease derived from the epithelium of the mammary glands. In 2012, breast cancer was ranked first in cancer incidence among women worldwide,

Correspondence to: Dr Zhe Liu, Oncology Center, West China Hospital, 37 Guoxuexiang, Wuhou, Chengdu, Sichuan 610041, P.R. China

E-mail: dragonsylvia@foxmail.com

Key words: microRNA, breast cancer, Akt/mTOR pathway, proliferation, invasion, migration with an estimated 1.7 million cases (1). For cancer-associated mortality, breast cancer was ranked the leading cause of death among women, with an estimated 521,900 mortalities worldwide in 2012 (1). It is therefore important to investigate potential targets for tumorigenesis and progression of breast cancer. Previous studies (2-4) have demonstrated that Akt/mTOR pathways appear to be a prime strategic target for breast cancer therapeutic development.

Akt and the mammalian target of rapamycin (mTOR) signaling pathways have regarded to serve critical roles in cell proliferation, motility, survival and therapy resistance (3). The activation of the Akt/mTOR pathway has been observed in a variety of tumors, including colon cancer, kidney cancer, some lymphomas, bone sarcoma and in particular, breast cancer (3). Inhibition of the Akt/mTOR pathway results in suppression of cell proliferation and promotion of cell death, making it an attractive target for oncology research (4).

MicroRNAs (miRs) are a type of multi-functional small non-coding RNA, which have numerous number and exists widely in animals and plants. In recent years, it has been demonstrated that miRs target mRNA at the transcription level and inhibit the mRNA translation process or promote the degradation of mRNA (5). A number of miRs have been reported to target or affect the Akt/mTOR pathways. Ectopic expression of miR-7, which has previously been identified as a tumor suppressor, inhibited tumor growth and metastasis, and restrained the expression of Akt, mTOR and the downstream P70S6K in hepatic cancer (6). In addition, it was demonstrated that mTOR and P70S6K are target genes of miR-7 (6). Other miRNAs including miR-144, miR-126, miR-199a-3p and miR-718, regulate the Akt/mTOR pathway in ovarian, oral, colorectal and human non-small-cell lung cancers and Kaposi's sarcoma (7-11). Few studies identified miRs regulating Akt/mTOR pathway in breast cancer (12-15). MiR-122 was observed to inhibit the cell proliferation and tumorigenesis of breast cancer and to suppress the activation of the Akt/mTOR pathway by targeting IGF-1R, which is an upstream molecule of the Akt/mTOR pathway (12). Another study identified three miRs (miR-147, miR-124, and miR-193a-3p) that inhibit cell-cycle progression and proliferation by targeting EGFR-driven cell-cycle network proteins in breast cancer (13). EGFR also serves a crucial role in the initiation of the Akt/mTOR pathway (14). Furthermore, 
miR-147 was found to repress cell invasion and proliferation, induce cell arrest at G1 in colon cancer (15). In addition, it has been shown that ectopic expression of miR-147 prevented Akt phosphorylation (15).

Although studies have identified miR-147 as a tumor suppressor $(14,15)$, the effect and the corresponding mechanisms of miR-147 in breast cancer remains to be investigated. Here, the present study aimed to understand the effect of miR-147 on the biological behaviors of breast cancer cells, phosphorylation of Akt and the potential downstream pathways regulating these processes in breast cancer.

\section{Materials and methods}

Cell culture. The normal mammary epithelial cell line MCF-10A and the breast cancer cell lines MCF7, SK-BR-3 and MDA-MB-231 were obtained from the Institute of Basic Medical Sciences of the Chinese Academy of Medical Sciences (Beijing, China). MCF-10A cells and MDA-MB-231 cells were cultured in Dulbecco's minimum essential medium (DMEM; Hyclone, Logan, Utah, USA) with 10\% fetal bovine serum (FBS; Lanzhou National Hyclone Bio-engineering, Lanzhou, China). MCF7 cells were cultured in RPMI 1640 (Hyclone) with 10\% FBS and SK-BR-3 cells were maintained in RPMI 1640+HEPES with 10\% FBS. All of the above cells were cultured at $37^{\circ} \mathrm{C}$ in a $5 \% \mathrm{CO}_{2}$ atmosphere incubator.

Reverse transcription-polymerase chain reaction (RT-PCR). Total RNA samples were isolated using Invitrogen TRIzol reagent (Thermofisher Scientific, Inc., Carlsbad, CA, USA) according to the manufacturer's instructions. Reverse transcription was then conducted using All-in-One miRNA qRT-PCR Detection kit (GeneCopoeia, Rockville, MD, USA) to obtain cDNA. The total reaction system was incubated at $42^{\circ} \mathrm{C}$ for $60 \mathrm{~min}$, then $70^{\circ} \mathrm{C}$ for $10 \mathrm{~min}$ and cooled on ice. The PCR reaction was performed using All-in-One miRNA qRT-PCR Detection kit (GeneCopoeia) with a Bio-Rad iCycler single-color real-time detection system (Bio-Rad Laboratories, Inc., Hercules, CA, USA). RNU6 was used as the internal control of miRNA. The primers for miR-147 and RNU6 were designed and sythesized by GeneCopoeia. The relative expression values of miRNA were calculated by the $2^{-\Delta \Delta C q}$ method. Cq is the intensity value of fluorescent signal detected by the thermal cycler. $\Delta \Delta \mathrm{Cq}=(\mathrm{CqmiRNA}-\mathrm{CqU} 6)$. Using $2^{-\Delta \Delta \mathrm{Cq}}$ as the expression of miR in each experimental group. The average expression level of MCF7 cells was set to 1 . The expression levels of the other cancerous groups were normalized to that of the MCF7 cell group, as the MCF7 cell group exibited the least intra-group difference with regard to miR-147 expression.

Ectopic expression of miR-147 and RNA interference. miR-147 mimic transfection was performed using mimics of miR147 or C. elegans miRNA (Guangzhou RiboBio Co., Ltd., Guangzhou, China) following the manufacturer's protocol. The final reagent concentration of the mimics was modulated to $50 \mathrm{nM}$. The si-Akt expressing plasmid vector was designed, synthesized and packaged by Shanghai GenePharma Co., Ltd (Shanghai, China). The quantity of si-Akt expressing plasmid vector used was $2 \mu \mathrm{g} /$ well ( 6 well plate). A total of $1.25 \mu \mathrm{l} / \mathrm{well}$ Invitrogen Lipo2000 (Thermofisher Scientific, Inc.) was used to transfect the miR mimic or siRNA into MDA-MB-231 cells at a density of $2.5 \times 10^{5}$ cells/well, according to the manufacturer's protocol. Cell proliferation assays were performed every $24 \mathrm{~h}$ for seven days after transfection. Total RNA and total protein were extracted two days after transfection, and the cells were dissociated two days after transfection for the Transwell assays.

Cell proliferation assay. A total of $2.5 \times 10^{3}$ cells/well were seeded into 96 -well plates with 5 repeated wells. The absorbance values were detected every $24 \mathrm{~h}$ for 7 days. Before the detection, $20 \mu \mathrm{l}$ MTT (Sigma-Aldrich, St. Louis, MO, USA) was added in each well and the cells were incubated for $4 \mathrm{~h}$. After incubation, the upper liquid was removed and $200 \mu$ l DMSO (Chengdu Kelong Chemical Co., Ltd., Chengdu, China) was added to each well. The absorbance value was determined at $490 \mathrm{~nm}$ using a Multiskan Spectrum microplate spectrophotometer (Thermofisher Scientific, Inc.).

Transwell assay. Boyden Well (BD Biosciences, Franklin Lakes, NJ, USA) was used for the assessment of the migration and invasion of transfected cells. Polycarbonate Microporous Membrane was placed between the upper and lower chambers. For the invasion assay, the membrane of the upper chambers were coated with $6 \mathrm{mg} / 1$ Matrigel (BD Biosciences) and incubated at $37^{\circ} \mathrm{C}$ for $30 \mathrm{~min}$. For the migration assay, the wells were not coated with Matrigel. MDA-MB-231 cells were seeded at a density of $1 \times 10^{5}$ cells in each upper chamber and DMEM+10\% FBS was placed in the lower chambers. The plates were then incubated at $37^{\circ} \mathrm{C}, 5 \% \mathrm{CO}_{2}$. After $24 \mathrm{~h}$, the membranes were removed and fixed in $4 \%$ paraformaldehyde (Chengdu Kelong Chemical Co., Ltd.), stained with Giemsa (Sigma-Aldrich) and washed with PBS buffer. The migration and invasion of each group were quantified as the mean number of cells found in ten microscope fields [magnification, x400; Eclipse E200; Nikon Instruments (Shanghai) Co., Ltd., Shanghai, China].

Western blot analysis. Cells were dissolved using RIPA buffer to obtain the cell lysates. Next, 5X Loading Buffer (Chengdu Cetme Science and Technology Co., Ltd., Chengdu, China) was added to the cell lysates, and incubated at $70^{\circ} \mathrm{C}$ for $10 \mathrm{~min}$. Total proteins were separated on 8-12\% gradient SDS-polyacrylamide gels and transferred to PVDF membranes (EMD Millipore, Billerica, MA, USA). $\beta$-actin was used as loading control. The PVDF membranes were then blocked in TBST with 5\% nonfat milk for $1 \mathrm{~h}$, and incubated with the following antibodies: monoclonal rabbit anti-human anti-Akt $(1: 1,000)$, anti-p-Akt $(1: 1,000)$, anti-P70S6K (1:1,000), anti-p-P70S6K $(1: 1,000)$, anti-4E-BP-1 (1:1,000), anti-p-4E-BP-1 (1:1,000) (CST, Boston, Massachusetts, USA) and monoclonal mouse anti-human anti- $\beta$-actin $(1: 1,000)$ (Santa Cruz Biotechnology, Dallas, Texas, USA). Following washing with TBST the membranes were incubated with polyclonal goat anti-rabbit or rabbit anti-mouse IgG secondary antibodies $(1: 10,000)$ (Beijing Zhongshan Golden Bridge Biotechnology Co., Ltd., Beijing, China) for $1 \mathrm{~h}$ then washed with TBST. The blots were developed using ECL reagents (EMD Millipore). The exposure and image capture was performed with a Gel Dox XR+ imaging system (Bio-Rad Laboratories, Inc.).

Statistical analysis. All quantitative data are expressed as the mean \pm standard error. The results were analyzed by Prism 6 


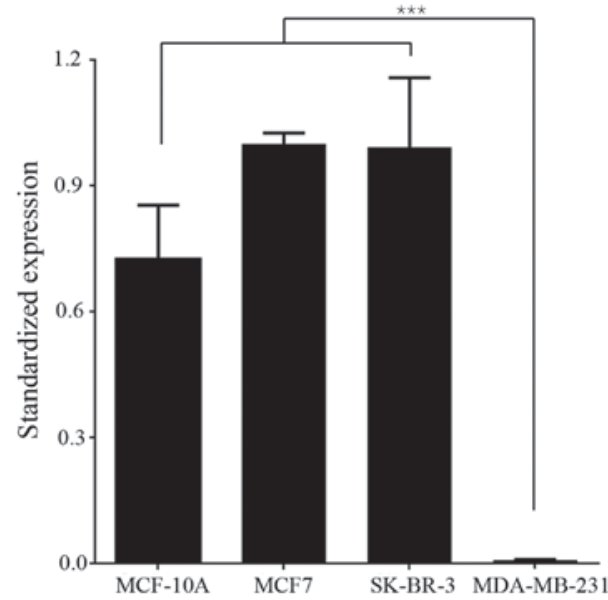

Figure 1. miR-147 expression was downregulated in the aggressive breast cancer cell line, MDA-MB-231 cells. Samples were normalized to RNU6. miR-147 expression in MDA-MB-231 cells was significantly reduced dompared with the other 3 cell types. ${ }^{* * *} \mathrm{P}<0.001$; all experiments were performed in triplicate.

software, version 6.01 (GraphPad Software, Inc., La Jolla, CA, USA). Statistical analyses were performed using Student's t-test for comparisons between each case group and the control group. Linear regression was used for cell growth curve comparisons. $\mathrm{P}<0.05$ was considered to indicate a statistically significant difference.

\section{Results}

miR-147 expression was downregulated in highly aggressive breast cancer cell line. To determine the function and mechanism of miR-147 in breast cancer, miR-147 expression was detected in breast cancer cell lines. Normal mammary epithelial cell line (MCF-10A), two less aggressive breast cancer cell lines (MCF7 and SK-BR-3), and a highly aggressive breast cancer cell line (MDA-MB-231) were cultured in vitro. The endogenous levels of miR-147 in the above cell lines were examined by RT-PCR. The level of miR-147 expression in MCF-10A, MCF7 and SK-BR-3 cells was relatively high, while the expression of miR-147 in MDA-MB-231 was too low to be detected. The difference in miR-147 expression level between MDA-MB-231 and the other 3 cell lines was statistically significant (Fig. 1; $\mathrm{P}<0.001$ ), indicating that the expression of miR-147 may be negatively correlated with the aggressiveness of breast cancer. The MDA-MB-231 cell line exhibits high aggressiveness and low miR-147 expression, and was chosen to be used in further experiments.

miR-147 repressed cell proliferation, invasion and migration. Proliferation, invasion and migration are examples of the characteristic aggressive properties of cancer cells. A 3-[4, 5-dimethylthiazol-2-yl]-2, 5-diphenyltetrazolium bromide (MTT) assay was used to investigate the effect of miR-147 on cell proliferation. MiR-147 was transiently transfected into the cell line MDA-MB-231 to form the 'miR-147' group. In the 'si-Akt' group, siRNA was transfected to knockdown the expression of Akt. Fig. 2 shows that there was a significant decrease in the MTT value in miR-147 transfected MDA-MB-231 cells on

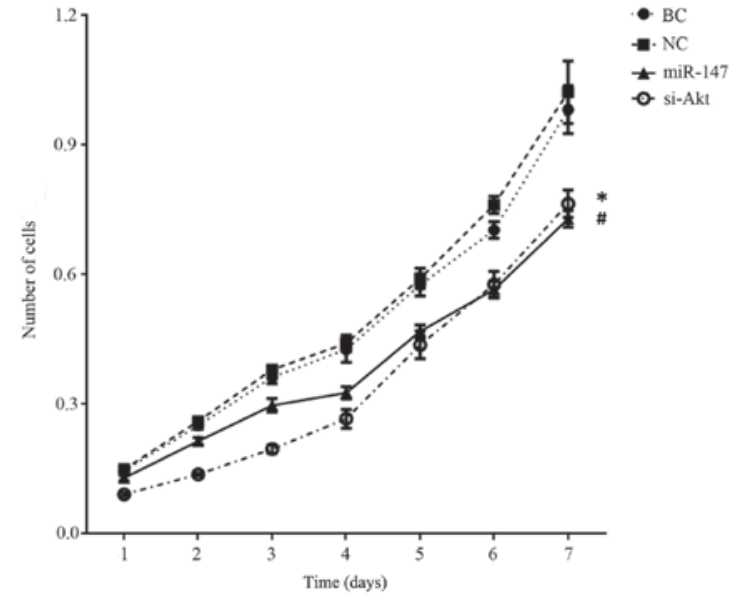

Figure 2. miR-147 repressed cell proliferation. ${ }^{\#} \mathrm{P}<0.001$ vs. BC and $\mathrm{NC}$; ${ }^{*} \mathrm{P}<0.001$ vs. BC. BC, blank control MDA-MB-231 cell; NC, MDA-MB-231 cell transfected with a $C$. elegans miRNA; miR-147, miR-147 mimics-transfected cells; si-Akt, specific siRNA knockdown of Akt.

day 7, in comparison to the blank control cells [MDA-MB-231 cells cultured in $\mathrm{DMEM} \pm 10 \%$ FBS; blank control, $(\mathrm{BC})]$ and negative control miR transfected cells $(\mathrm{P}<0.001)$. In addition, in comparison with the $\mathrm{BC}$ cells, the knockdown of Akt in MDA-MB-231 cells also resulted in a reduced MTT value $(\mathrm{P}<0.001)$. Although miR-147 transfected cells had a higher MTT value compared with knockdown of Akt cells on day 4 $(\mathrm{P}=0.007)$, a statistically significant difference was not observed between the MTT value of the miR-147 group and si-Akt group on overall trend $(\mathrm{P}=0.086)$.

Transwell assays were used to measure the cell invasive and migrating capability. The number of cells that invaded the Matrigel or traversed the microporous membrane are presented in Fig. 3. The number of invasive and migratory miR-147 transfected cells was significantly less than the BC and miR transfected negative control cells both in the invasion assay $(\mathrm{P}<0.01$; Fig. $3 \mathrm{~A}$ and $\mathrm{B})$ and migration assay $(\mathrm{P}<0.001)$; Fig. $3 \mathrm{C}$ and D). Similarly, the number of invasive and migratory cells following the knockdown of Akt was less than the $\mathrm{BC}$ cells both in invasion assay $(\mathrm{P}<0.001$; Fig. $3 \mathrm{~A}$ and $\mathrm{B})$ and migration assay $(\mathrm{P}<0.001$; Fig. $3 \mathrm{C}$ and $\mathrm{D})$. The number of invasive cells following knockdown of Akt was less than the number of invasive miR-147 transfected cells in the invasion assay ( $\mathrm{P}=0.047)$, however no significant difference between the two in the number of migrating cells was observed in the migration assay $(\mathrm{P}=0.769)$.

miR-147 reduced the activation of Akt/mTOR signaling pathway. Previous studies have reported that Akt may be a potential target of miR-147 $(13,15)$. To investigate how miR-147 affects AKT, and the role of Akt in miR-147 induced cell proliferation, invasion and migration inhibition, western blotting was performed to assess the expression and phosphorylation of the Akt protein and the downstream key effectors in the Akt/mTOR pathway. Fig. 4 demonstrates that miR-147 expression repressed the phosphorylation of Akt, P70S6k and 4E-BP-1 in MDA-MB-231 cell, but appeared to have no effect on the total level of these proteins. Knockdown of Akt restrained both Akt phosphorylation and total Akt level, and 
A

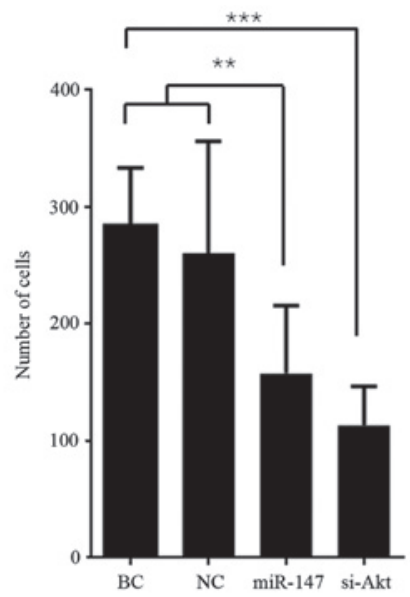

C

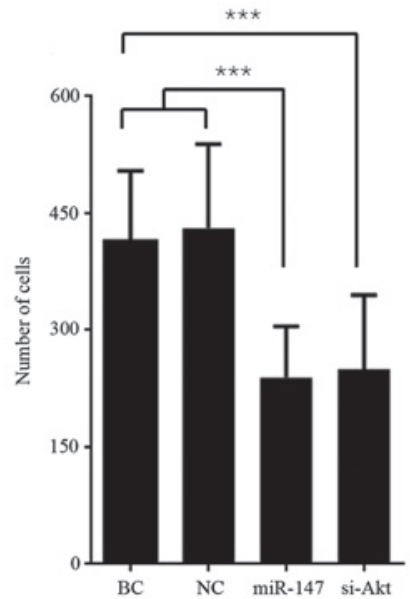

B
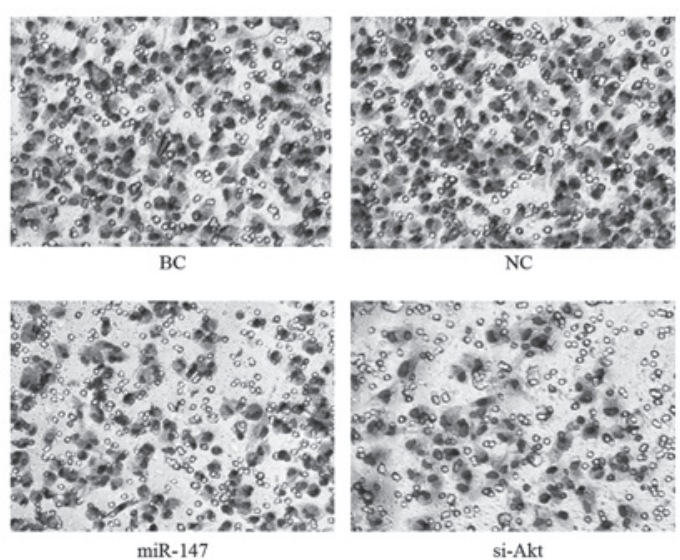

D
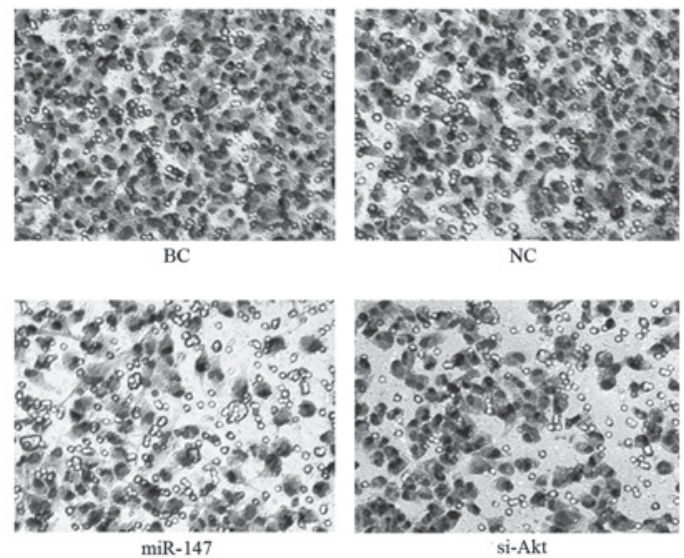

Figure 3. miR-147 repressed cell invasion and migration. (A) Number of cells that invaded the Matrigel and membrane. ${ }^{* *} \mathrm{P}<0.01$ vs. $\mathrm{BC}$ and $\mathrm{NC}$; ${ }^{* * *} \mathrm{P}<0.001 \mathrm{vs}$. BC. (B) Typical images of the invasion assay, at $\mathrm{x} 400$ magnification. (C) Number of cells that traversed the membrane. ${ }^{* * *} \mathrm{P}<0.001 \mathrm{vs}$. $\mathrm{BC}$ and $\mathrm{NC}$; ${ }^{* * *} \mathrm{P}<0.001 \mathrm{vs}$. BC. (D) Typical images of the migration assay, at x400 magnification. BC, blank control cells; NC, normal control cells; miR-147, miR-147 mimics-transfected cells; si-Akt, specific siRNA knockdown of Akt.

the downstream phosphorylation of P70S6k and 4E-BP-1. The total P70S6K level was slightly upregulated in the 'si-Akt' group. Generally, the effect of miR-147 on Akt/mTOR pathway was analogous to that of siRNA directed at Akt.

\section{Discussion}

The present study demonstrated that miR-147 is relatively highly expressed in the normal breast epithelial cell line (MCF-10A) and the less aggressive breast cancer cell lines (MCF7, SK-BR-3) and markedly low expression in the most aggressive breast cancer cell line studied (MDA-MB-231). Few studies have reported the expression of miR-147 in breast cancer. In respect to other types of tumor, Yao et al (16) demonstrated that miR-147 was overexpressed in gastric cancer compared with normal gastric tissue; Wong et al (17) reported that miR-147 has higher expression in squamous cell carcinoma of the tongue compared with paired normal tissue. miR-147 expression was previously demonstrated to be higher in recurrence hepatocellular carcinoma following liver transplantation when compared with non-recurrence hepatocellular carcinoma (18). The result of the present study is inconsistent with the previous studies, which may be due to following reasons. First, the tumors in the previous studies are different types to the current study, and may result in the differences observed in its detection. In addition, the above studies analyzed the expression of miR-147 by microarray, which is high throughput but lower in accuracy (16-18). Yao et al (16) and Wong et al (17) did not verify miR-147 expression by PCR, in comparison to the present study that used RT-PCR to accurately quantify miR levels. The present study demonstrated that miR-147 was expressed at higher levels in MCF7 and SK-BR-3 cell lines compared with in MCF-10A cells $(\mathrm{P}<0.05)$ : This result suggests that less aggressive breast cancers may express higher levels of miR-147 than that of normal tissue, while miR-147 expression is significantly reduced in more aggressive breast cancers. Equally, other types of tumors may also express high levels of miR-147, as was indicated in the above studies $(16,17)$. However, the association between miR-147 expression and the grade malignancy of breast cancer remains to be defined.

Akt/mTOR signaling pathway is considered to be one of the most frequently aberrantly activated pathways, which affects $30-50 \%$ of human tumors (3). Akt is a key regulator of survival during cellular stress, which appears to be crucial in cancer (19). mTOR is a serine/threonine kinase widely expressed in mammalian cells. Through its downstream effectors, P70S6K and $4 \mathrm{E}-\mathrm{BP}-1$, it is involved in the initiation of ribosomal translation of mRNA into proteins necessary for cell proliferation, cell 


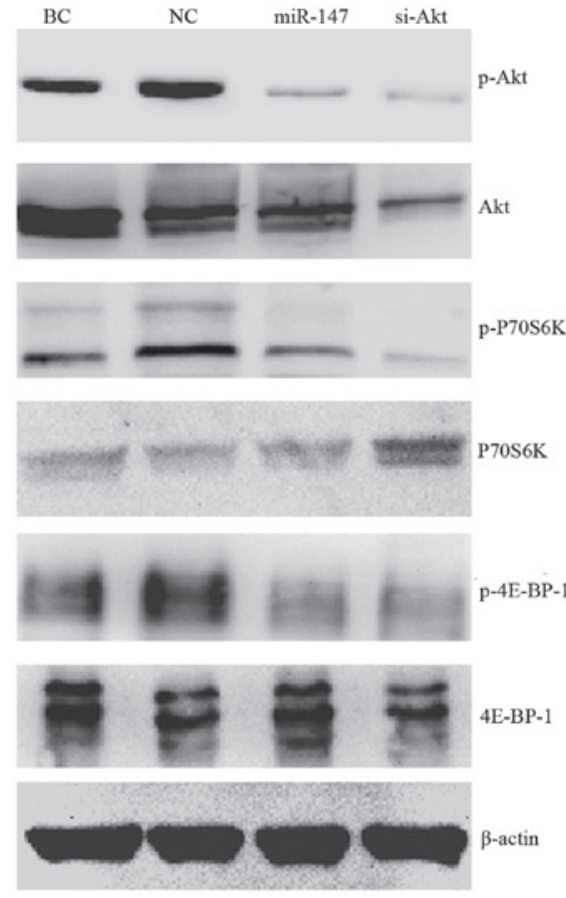

Figure 4. miR-147 inhibited the activation of the Akt/mTOR signaling pathway. $\beta$-Actin was used as loading control. Ectopic expression of miR-147 downregulated Akt phosphorylation (p-Akt), P70S6K phosphorylation (p-P70S6K) and 4E-BP-1 phosphorylation (p-4E-BP-1), compared with blank control MDA-MB-231 cells (BC) and negative control miR-transfected cells (NC). Knockdown of Akt (si-Akt) reduced phosphorylation and the total level of Akt. The phosphorylation of P70S6K and 4E-BP-1 was also inhibited in the si-Akt group. The total P70S6K level was slightly upregulated in the si-Akt group.

metabolism and cell cycle progression (20). Akt and the downstream P70S6K and 4E-BP-1 serve key roles in Akt/mTOR pathway. The phosphorylation of these molecules represents the activation of Akt/mTOR pathway. On the contrary, reduced phosphorylation of Akt, P70S6K and 4E-BP-1 indicates suppression of the pathway (21). The present study demonstrated that ectopic expression of miR-147 restrained the phosphorylation of Akt and the downstream P70S6K and 4E-BP-1. This effect was similar to that of siRNA which specifically silence the expression of Akt. The result indicates that miR-147 may serve a suppressor role in Akt/mTOR pathway. A previous study reported that overexpression of miR-147 may downregulate the phosphorylation of Akt in colon cancer (15). In addition, Uhlmann et al (13) even identified Akt2 and CyclinD1 as direct targets of miR-147. However, these two studies did not demonstrate the effect of miR-147 in the downstream mTOR pathway. The present study reported the association between miR-147 and the Akt/mTOR pathway in breast cancer.

The current study demonstrated that miR-147 expression represses the cell proliferation, invasion and migration in breast cancer, similarly to the effect of siRNA silencing Akt expression. This result is consistent with Uhlmann et al (13). miR-147 was also demonstrated to inhibit the invasion and motility of colon cancer cells (15). The comparison between miR-147 and knockdown of Akt with siRNA was not conducted in the above studies. The present study directly compared the effect of miR-147 with that of siRNA downregulating Akt, and confirmed that the suppressor function of miR-147 was achieved through Akt/mTOR pathway. miR-147 may provide a novel target for breast cancer therapy targeting Akt/mTOR pathway.

The endogenous level of miR-147 was downregulated in highly aggressive breast cancer cell line. Ectopic expression of miR-147 repressed cell proliferation, invasion and migration by inhibiting activation of Akt/mTOR signaling pathway in breast cancer cell. The above effects of miR-147 were analogous to that of siRNA targeted to specifically silence Akt. Acting as a potential tumor suppressor, miR-147 indicates a novel avenue for breast cancer therapy targeting Akt/mTOR pathway.

\section{Acknowledgements}

The authors sincerely thank Dr Chen Nianyong for comments, improvement and submission on the manuscript; and Dr Wang Yanping and Dr Wang Zhu of Tumor Molecular Diagnostic Laboratory for their technical support.

\section{References}

1. Torre LA, Bray F, Siegel RL, Ferlay J, Lortet-Tieulent J and Jemal A: Global cancer statistics, 2012. CA Cancer J Clin 65: 87-108, 2015.

2. Mita MM, Mita A and Rowinsky EK: Mammalian target of rapamycin: A new molecular target for breast cancer. Clin Breast Cancer 4: 126-137, 2003.

3. Samuels Y, Wang Z, Bardelli A, Silliman N, Ptak J, Szabo S, Yan H, Gazdar A, Powell SM, Riggins GJ, et al: High frequency of mutations of the PIK3CA gene in human cancers. Science 304: $554,2004$.

4. Hennessy BT, Smith DL, Ram PT, Lu Y and Mills GB: Exploiting the PI3K/AKT pathway for cancer drug discovery. Nat Rev Drug Discov 4: 988-1004, 2005.

5. Bartel DP: MicroRNAs: Genomics, biogenesis, mechanism, and function. Cell 116: 281-297, 2004.

6. Fang Y, Xue JL, Shen Q, Chen J and Tian L: MicroRNA-7 inhibits tumor growth and metastasis by targeting the phosphoinositide 3-kinase/Akt pathway in hepatocellular carcinoma. Hepatology 55: 1852-1862, 2012.

7. Nagaraja AK, Creighton CJ, Yu Z, Zhu H, Gunaratne PH, Reid JG, Olokpa E, Itamochi H, Ueno NT, Hawkins SM, et al: A link between mir-100 and FRAP1/mTOR in clear cell ovarian cancer. Mol Endocrinol 24: 447-463, 2010.

8. Uesugi A, Kozaki K, Tsuruta T, Furuta M, Morita K, Imoto I, Omura $\mathrm{K}$ and Inazawa J: The tumor suppressive microRNA miR-218 targets the mTOR component Rictor and inhibits AKT phosphorylation in oral cancer. Cancer Res 71: 5765-5778, 2011.

9. Iwaya T, Yokobori T, Nishida N, Kogo R, Sudo T, Tanaka F, Shibata K, Sawada G, Takahashi Y, Ishibashi M, et al: Downregulation of miR-144 is associated with colorectal cancer progression via activation of mTOR signaling pathway. Carcinogenesis 33: 2391-2397, 2012.

10. Yu T, Li J, Yan M, Liu L, Lin H, Zhao F, Sun L, Zhang Y, Cui Y, Zhang F, et al: MicroRNA-193a-3p and -5p suppress the metastasis of human non-small-cell lung cancer by downregulating the ERBB4/PIK3R3/mTOR/S6K2 signaling pathway. Oncogene 34: 413-423, 2015.

11. Xue M, Yao S, Hu M, Li W, Hao T, Zhou F, Zhu X, Lu H, Qin D, Yan Q, et al: HIV-1 Nef and KSHV oncogene K1 synergistically promote angiogenesis by inducing cellular miR-718 to regulate the PTEN/AKT/mTOR signaling pathway. Nucleic Acids Res 42: 9862-9879, 2014.

12. Wang B, Wang $H$ and Yang Z: MiR-122 inhibits cell proliferation and tumorigenesis of breast cancer by targeting IGF1R. PLoS One 7: e47053, 2012.

13. Uhlmann S, Mannsperger H, Zhang JD, Horvat EÁ, Schmidt C, Küblbeck M, Henjes F, Ward A, Tschulena U, Zweig K, et al: Global microRNA level regulation of EGFR-driven cell-cycle protein network in breast cancer. Mol Syst Biol 8: 570, 2012.

14. Lo HW, Hsu SC and Hung MC: EGFR signaling pathway in breast cancers: From traditional signal transduction to direct nuclear translocalization. Breast Cancer Res Treat 95: 211-218, 2006. 
15. Lee CG, McCarthy S, Gruidl M, Timme C and Yeatman TJ: MicroRNA-147 induces a mesenchymal-to-epithelial transition (MET) and reverses EGFR inhibitor resistance. PLoS One 9: e84597, 2014

16. Yao Y, Suo AL, Li ZF, et al: MicroRNA profiling of human gastric cancer. Mol Med Rep 2: 963-970, 2009.

17. Wong TS, Liu XB, Wong BY, Ng RW, Yuen AP and Wei WI: Mature miR-184 as Potential Oncogenic microRNA of Squamous Cell Carcinoma of Tongue. Clin Cancer Res 14: 2588-2592, 2008.
18. Han ZB,ZhongL, Teng MJ, et al: Identification of recurrence-related microRNAs in hepatocellular carcinoma following liver transplantation. Mol Oncol 6: 445-457, 2012.

19. Datta SR, Brunet A and Greenberg ME: Cellular survival: A play in three Akts. Genes Dev 13: 2905-2927, 1999.

20. Sehgal SN, Baker H and Vézina C: Rapamycin (AY-22,989), a new antifungal antibiotic. II. Fermentation, isolation and characterization. J Antibiot (Tokyo) 28: 727-732, 1975.

21. Morgensztern D and McLeod HL: PI3K/Akt/mTOR pathway as a target for cancer therapy. Anticancer Drugs 16: 797-803, 2005. 